\title{
The column density towards LMC X-1
}

\author{
M. Hanke ${ }^{1}$, J. Wilms ${ }^{1}$, M. A. Nowak ${ }^{2}$, L. Barragán ${ }^{1}$, and N. S. Schulz ${ }^{2}$ \\ 1 Dr. Karl Remeis-Observatory \& ECAP, University of Erlangen-Nuremberg, Sternwartstr. 7, 96049 Bamberg, Germany \\ e-mail: Manfred.Hanke@sternwarte.uni-erlangen.de \\ 2 MIT Kavli Institute for Astrophysics and Space Research, NE80-6077, 77 Mass. Ave., Cambridge, MA 02139, USA \\ Received 2 November 2009 / Accepted 17 December 2009
}

ABSTRACT

\begin{abstract}
We measure the neutral absorption towards the black hole X-ray binary system LMC X-1 from six archival soft X-ray spectra obtained with the gratings and/or CCD detectors on Chandra, XMM-Newton, and Swift. Four spectral models for the soft continuum are investigated. While the powerlaw model may overestimate $N_{\mathrm{H}}$ considerably, the others give consistent results. Taking the lower metalicity of the Large Magellanic Cloud into account, we find equivalent hydrogen column densities of $N_{\mathrm{H}}=(1.0-1.3) \times 10^{22} \mathrm{~cm}^{-2}$, with a systematic dependence on the orbital phase. This variation in the neutral absorption can nearly explain the orbital modulation of the soft X-ray flux recently detected with the All Sky Monitor (ASM) on the Rossi X-ray Timing Explorer (RXTE).
\end{abstract}

Key words. X-rays: individuals: LMC X-1 - X-rays: binaries - X-rays: ISM - galaxies: abundances

\section{Introduction}

The extragalactic X-ray sources in the Large Magellanic Cloud (LMC), our $\approx 48 \mathrm{kpc}$ distant neighboring galaxy, were discovered in the late 1960s (Mark et al. 1969; Price et al. 1971). Because the density of stars is high, their optical identifications were uncertain for a long time. The X-ray binary system (XRB) LMC X-1 is located $\approx 0.5$ south-southeast of the 30 Doradus star-formation region, in the NGC 2078 (LMC N159F) nebula. Pakull (1980), Hutchings et al. (1983, 1987), and Cowley et al. (1995) were able to identify the counterpart of LMC X-1 with an $m_{V}=14$ m 5 O7/8 giant (Hutchings et al. 1983; Negueruela \& Coe 2002). This has allowed the placement of strong dynamical constraints on the compact object's mass. Orosz et al. (2009) have recently used optical spectra of this star - labeled as "star \#32" by Cowley et al. (1978) and also often called Pakull's star to confirm the black hole $(\mathrm{BH})$ candidacy of LMC X-1. They derive an orbital period of $3.909 \mathrm{~d}$, which is consistent with the modulation of the soft X-ray flux of LMC X-1 (Levine \& Corbet 2006). Deriving an extinction of $A_{V}=2.28 \pm 0.06-$ much more than previously assumed - from the $V-K$ color excess, Orosz et al. (2009) infer a BH mass of $10.9 \pm 1.6 M_{\odot}$.

The persistent XRB LMC X-1 is the only dynamically confirmed $\mathrm{BH}$ candidate that so far has only been found in the high/soft (thermal dominant) X-ray spectral state; that is, its $\mathrm{X}$-ray spectrum can be described by a multi-temperature disk blackbody component plus a weak soft $(\Gamma \gg 2)$ power-law component (Ebisawa et al. 1989; Schlegel et al. 1994; Wilms et al. 2001; Nowak et al. 2001; Haardt et al. 2001; Cui et al. 2002; Yao et al. 2005). In comparison, LMC X-3 usually shows a similarly soft X-ray spectrum, but also (partial) transitions to the low/hard state (Wilms et al. 2001; Smith et al. 2007), while Cyg X-1 regularly transits between the low/hard and a soft-intermediate state and never reaches the thermal dominant state (Wilms et al. 2006). LMC X-1 is therefore an ideal target for measuring the $\mathrm{BH}$ spin parameter $a_{*}$ from the soft X-ray continuum produced by the relativistic accretion disk. Gierliński et al. (2001) constrained $a_{*}$ to be less than 0.998 from a $24 \mathrm{ks} 0.7-10 \mathrm{keV}$ ASCA-SIS spectrum. Gou et al. (2009) have recently reported $a_{*}=0.90_{-0.09}^{+0.04}$ from 18 selected $R X T E$-PCA spectra with exposures between 5-11 ks and covering 2.5-20 keV. These authors fix the column density for the photoelectric absorption to $N_{\mathrm{H}}=$ $4.6 \times 10^{21} \mathrm{~cm}^{-2}$ as reported by Cui et al. (2002) based on low statistics.

An appropriate description of the absorption is, however, indispensable for modeling the soft X-ray continuum and likewise for modeling the visual extinction, hence the derivation of the system parameters from the dereddened optical spectrum of the companion star. In this Letter, we therefore aim to accurately describe the column density towards LMC X-1. We describe the data in Sect. 2 and present the methods and our analysis in Sect. 3. We summarize and discuss our results in Sect. 4.

\section{Observations and data reduction}

We study the spectra from all six recent observations with instruments providing soft X-ray spectra (Table 1).

The Chandra observation $\mathrm{C} 1$ was performed using the HETGS (Canizares et al. 2005) and with the detector CCDs operated in timed exposure mode. The \pm first order HEG and MEG spectra, as well as the corresponding response matrices, were taken from the Chandra Transmission Grating Catalog archive TGCat ${ }^{1}$.

All instruments of XMM-Newton (Jansen et al. 2001) were active during the first (shorter) $X M M$ observation X1. The EPICpn camera (Strüder et al. 2001) was operated in timing mode. Its data are therefore not affected by photon pile-up (Wilms et al. 2003). The same is true for data from the Reflection Grating Spectrometers (RGS; den Herder et al. 2001) due to their dispersion of the photons, but not for data from the MOS cameras (Turner et al. 2001), which were operated in full frame imaging mode. For this reason, we only use the EPIC-pn spectrum and

\footnotetext{
1 See http://tgcat.mit.edu
} 
A\&A 509, L8 (2010)

Table 1. Log of recent soft X-ray observations of LMC X-1 with good S/N. (Instruments not considered here are in parenthesis.)

\begin{tabular}{ccccccccc}
\hline \hline Obs. & Start date & $\begin{array}{c}\text { Start date } \\
(\mathrm{MJD})\end{array}$ & $\begin{array}{c}\text { Exposure } \\
(\mathrm{ks})\end{array}$ & $\phi_{\mathrm{orb}}^{(\mathrm{T3})}$ & $\phi_{\mathrm{orb}}^{(\mathrm{F9})}$ & Satellite & ObsID & Instruments \\
\hline C1 & $2000-01-16$ & 51559.2 & 19 & $0.45-0.51$ & $0.50-0.56$ & Chandra & 93 & HETGS \\
X1 & $2000-10-21$ & 51838.7 & $5-7$ & $0.94-0.96$ & $0.98-0.01$ & XMM & 0112900101 & PN, RGS 1+2, (MOS 1+2) \\
X2 & $2002-09-26$ & 52543.2 & 35 & $0.17-0.28$ & $0.21-0.32$ & XMM & 0023940401 & RGS 1+2, (MOS 1+2) \\
S1 & $2007-10-31$ & 54404.7 & 2.4 & $0.37-0.42$ & $0.37-0.43$ & Swift & 00037079001 & (BAT), XRT/PC, (UVOT) \\
S2 & $2007-12-06$ & 54440.4 & 9.8 & $0.49-0.61$ & $0.50-0.62$ & Swift & 00037079002 & (BAT), XRT/WT, (UVOT) \\
S3 & $2007-12-10$ & 54444.1 & 4.4 & $0.43-0.50$ & $0.43-0.50$ & Swift & 00037079003 & (BAT), XRT/WT, (UVOT) \\
\hline
\end{tabular}

Notes. ${ }^{(\dagger)}$ Orbital phase calculated from the ephemeris of Orosz et al. (2009, Table 3): $T_{0}=$ MJD 53 390.8436, $P=3.90917 \mathrm{~d}$; ${ }^{(\$)}$ orbital phase calculated from the ephemeris of Orosz et al. (2009, Fig. 9): $T_{0}=$ MJD $53390.75174, P=3.9094 \mathrm{~d}$.

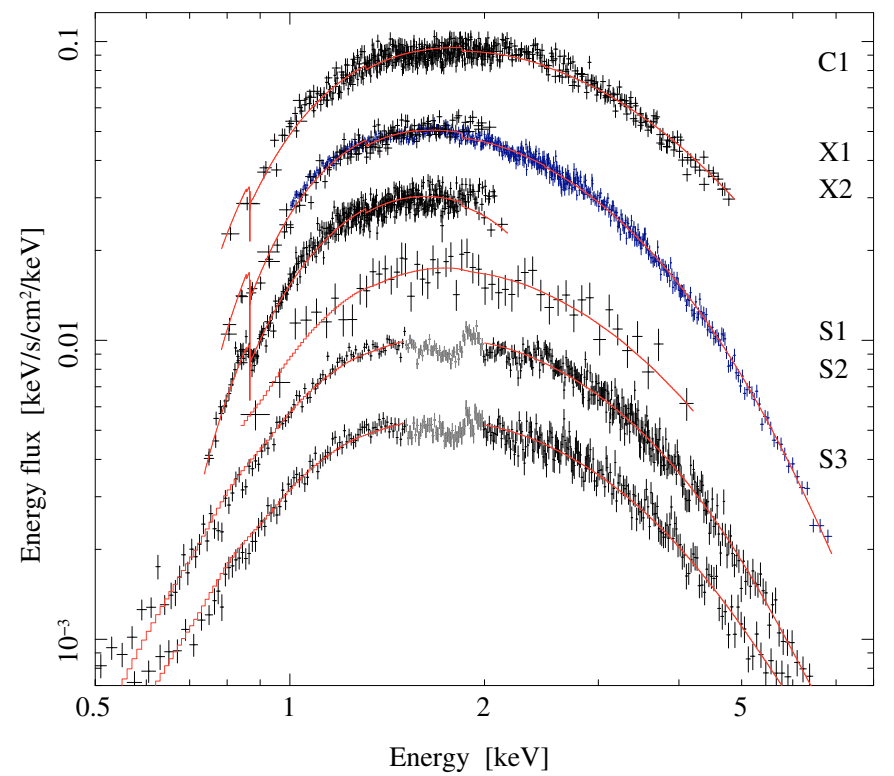

Fig. 1. Flux-corrected spectra of LMC X-1 from the six observations, shifted in flux according to the labels with respect to $\mathrm{C} 1$ for visual clarity. The gray data have been ignored because of calibration issues. The models shown here for illustrative purposes are also broadened by the instrumental response.

the first and second order spectra of RGS 1 and 2. For the second (longer) $X M M$ observation $\mathrm{X} 2$, however, no pn-data are available. The data were reduced with the Science Analysis Software, XMMSAS, v. 7.1, following standard procedures, i.e., applying the SAS tasks epchain, emchain, rgsproc, evselect, rmfgen, and arfgen to produce spectra and response matrices.

Swift's X-ray telescope (XRT; Burrows et al. 2005) was operated in photon counting (PC) mode during the first Swift observation $\mathrm{S} 1$, which resulted in pile-up. For S2 and S3, the windowed timing (WT) mode was used. After reprocessing the data to apply the newest calibration, spectra were extracted using standard FTOOLS, handled via xselect. For the PC mode observation S1, we extract an annulus to exclude the region affected by pile-up, only yielding a low-quality spectrum. Ancillary response files were created with xrtmkarf, and suitable response files for each observation were obtained from the CALDB. The WT mode spectra are not calibrated as well as the PC mode one around the Si edge (Fig. 1), so we exclude their $1.5-2 \mathrm{keV}$ data.

All spectral analysis was performed with the Interactive Spectral Interpretation System (ISIS; Houck \& Denicola 2000; Noble et al. 2006; Noble \& Nowak 2008) ${ }^{2}$.

\footnotetext{
${ }^{2}$ See http://space.mit.edu/cxc/isis/
}

Table 2. Comparison of elemental abundances (by number) in the Galactic ISM and in the LMC as $\epsilon(X)=12+\log _{10}(X / \mathrm{H})$.

\begin{tabular}{cccc}
\hline \hline$X$ & $\epsilon_{\mathrm{gal}}(X)^{(1)}$ & $\epsilon_{\mathrm{LMC}}(X)$ & $10^{\Delta \epsilon(X)}$ \\
\hline $\mathrm{He}$ & 10.99 & $10.93^{(5)}$ & 0.87 \\
$\mathrm{C}$ & 8.38 & $8.03^{(2)}$ & 0.45 \\
$\mathrm{~N}$ & 7.88 & $7.01^{(2)}$ & 0.13 \\
$\mathrm{O}$ & 8.69 & $8.38^{(2)}$ & 0.49 \\
$\mathrm{Ne}$ & 7.94 & $7.6^{(4)}$ & 0.46 \\
$\mathrm{Mg}$ & 7.40 & $7.12^{(2)}$ & 0.53 \\
$\mathrm{Si}$ & 7.27 & $7.21^{(2)}$ & 0.87 \\
$\mathrm{~S}$ & 7.09 & $6.7^{(4)}$ & 0.41 \\
$\mathrm{Ar}$ & 6.41 & $6.2^{(4)}$ & 0.62 \\
$\mathrm{Fe}$ & 7.43 & $7.2^{(3)}$ & 0.59 \\
\hline
\end{tabular}

References. (1) Wilms et al. (2000) or using xspec_abund("wilm”); in ISIS; (2) Przybilla (priv. comm.): average of 7 B-stars in the LMC (see also Korn et al. 2002, 2005); (3) Przybilla (priv. comm.): 1 star in the LMC (see also Przybilla et al. 2008); (4) Garnett (1999): H II regions in the LMC; (5) Dufour (1984).

Notes. The last column is the LMC abundance relative to the Galactic abundance, which is a parameter of the tbvarabs absorption model (Wilms et al. 2000, 2009, in prep.). For all other elements (which hardly contribute to the absorption in the soft X-ray band), the average value $10^{\Delta \epsilon(X)}=0.5$ is assumed.

\section{Analysis}

An overview of previous $N_{\mathrm{H}}$ measurements for LMC X-1 is given by Orosz et al. (2009, Table 2). We caution, however, that only $<12 \%$ of the hydrogen column density towards the LMC, $N_{\mathrm{H}}=4 \times 10^{21} \mathrm{~cm}^{-2}$ (measured in the LAB $21 \mathrm{~cm}$ survey; Kalberla et al. 2005; Bajaja et al. 2005), is of Galactic origin ${ }^{3}$, while the largest part is detected at $v_{\mathrm{LSR}}=200-300 \mathrm{~km} \mathrm{~s}^{-1}$ and thus is probably local to the LMC (Richter et al. 1987). As the absorption in the $0.5-10 \mathrm{keV}$ band is mostly caused by metals (Wilms et al. 2000) and the LMC has a much lower metallicity than our Galaxy, we compile both abundance sets in Table 2. The LMC abundances are henceforth used throughout our analysis.

As for all previous observations (see Sect. 1), the X-ray spectra of LMC X-1 investigated here are very soft (see Fig. 1), but a hard (albeit very steep) component in addition to a thermal one is nonetheless needed to describe the data, except for S2 and S3. The powerlaw model, however, becomes unphysically strong at low energies (e.g., Shrader \& Titarchuk 1998; Done et al. 2002). A steep photon index $\Gamma \gg 2$ (e.g., $\Gamma=3.7 \pm$ 0.1 as measured for $\mathrm{X} 1$, which has the best high-energy coverage

\footnotetext{
3 See http://www.astro.uni-bonn.de/ webaiub/english/ tools_labsearch.php?alpha $=05+39+38.7 \&$ beta $=-69+44+36$
} 
Table 3. Column density in units of $10^{22} \mathrm{~cm}^{-2}$ for the six observations and the sine fit, obtained with different continuum models.

\begin{tabular}{cccccccc}
\hline \hline $\begin{array}{c}\text { Observation or fit } \\
\phi_{\text {orb }}^{(\mathrm{T3})}\end{array}$ & $\begin{array}{c}\mathrm{X} 2 \\
0.17-0.28\end{array}$ & $\begin{array}{c}\mathrm{S} 1 \\
0.37-0.42\end{array}$ & $\begin{array}{c}\mathrm{S} 3 \\
0.43-0.50\end{array}$ & $\begin{array}{c}\mathrm{C} 1 \\
0.45-0.51\end{array}$ & $\begin{array}{c}\mathrm{S} 2 \\
0.49-0.61\end{array}$ & $\begin{array}{c}\text { X1 } \\
0.94-0.96\end{array}$ & $\begin{array}{c}\text { Sine fit } \\
\text { full orbit }\end{array}$ \\
\hline diskbb + powerlaw & $\left(2.00_{-0.19}^{+0.17}\right)^{*}$ & $\left(1.2_{-0.2}^{+0.5}\right)^{*}$ & $0.96_{-0.02}^{+0.03}$ & $\left(1.25_{-0.01}^{+0.04}\right)^{*}$ & $1.031 \pm 0.017$ & $\left(1.81_{-0.05}^{+0.06}\right)^{*}$ & $(1.43 \pm 0.43)^{*}$ \\
eqpair & $1.279 \pm 0.005$ & $1.17_{-0.10}^{+0.15}$ & $1.02 \pm 0.02$ & $1.065_{-0.019}^{+0.000}$ & $1.088 \pm 0.017$ & $1.191_{-0.007}^{+0.006}$ & $1.15 \pm 0.15$ \\
simpl(kerrbb) & $1.278 \pm 0.005$ & $1.17_{-0.10}^{+0.11}$ & $1.01_{-0.02}^{+0.03}$ & $1.085_{-0.016}^{+0.018}$ & $1.088 \pm 0.017$ & $1.187_{-0.012}^{+0.014}$ & $1.15 \pm 0.14$ \\
simpl(diskbb) & $1.288 \pm 0.016^{\dagger}$ & $1.14_{-0.11}^{+0.15}$ & $0.97 \pm 0.02$ & $1.009_{-0.017}^{+0.018}$ & $1.038 \pm 0.017$ & $1.133_{-0.004}^{+0.005}$ & $1.10 \pm 0.18$ \\
\hline
\end{tabular}

Notes. Quoted errors are statistical uncertainties at the $90 \%$ confidence level for the observations, but semi-amplitudes for the sine fits.

(*) The diskbb + powerlaw model overestimates $N_{\mathrm{H}}$ more, the more the powerlaw contributes at low energies, see text.

(†) As the lack of data above $2 \mathrm{keV}$ did not allow us to constrain the power law with the simpl model, we only used diskbb.

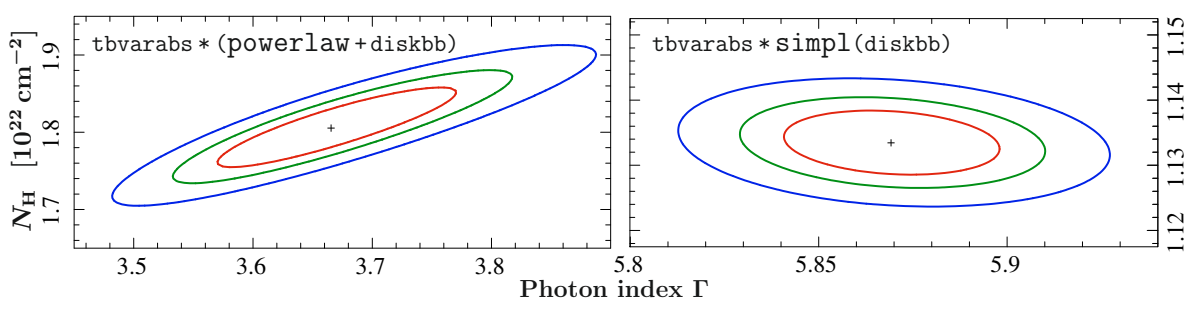

Fig. 2. Correlation of the column density $N_{\mathrm{H}}$ and the photon index $\Gamma$, derived with the powerlaw model (left) and the simpl model (right) for observation $\mathrm{X} 1$. The contours show the $68 \%, 90 \%$, and $99 \%$ confidence regions for two parameters of interest (i.e., $\Delta \chi^{2}=2.30$, 4.61, and 9.21). due to the EPIC-pn spectrum) is compensated for in spectral fits by an incorrectly strong absorption (e.g., Yao et al. 2005; Suchy et al. 2008; Gou et al. 2009). In contrast, the empirical convolution model simpl (Steiner et al. 2009) has an intrinsic lowenergy cut-off when convolving an input spectrum modeled by, e.g., diskbb or kerrbb ${ }^{4}$. Figure 2 shows that the (well known) correlation between $N_{\mathrm{H}}$ and $\Gamma$ vanishes when simpl is used instead of powerlaw. Although an even steeper photon index was found using simpl, the value of $N_{\mathrm{H}}$ is lower and is more narrowly constrained.

Because the derived absorption might depend on the shape of the continuum, we investigated different models, namely empirical ones - such as diskbb + powerlaw, simpl (diskbb), and simpl(kerrbb) (Li et al. 2005) - and the physical Comptonization model eqpair (Coppi 2000) ${ }^{5}$. These models typically describe our data equally well. In all fits, the disk has a temperature between 0.65 and $1.1 \mathrm{keV}$. The other parameters, too, are similar to previously obtained values. Table 3 and Fig. 3 show our results for the column density (assuming the LMC abundances given in Table 2) as a function of orbital phase $\phi_{\text {orb }}$ for each of the six observations and all four of the aforementioned continuum models. In all cases where a steep power law substantially contributes to the model, the diskbb + powerlaw model gives a much higher $N_{\mathrm{H}}$ than the other models, because of the systematic error of the powerlaw model. We therefore ignore these values. The other models, however, are quite consistent with one another: their agreement on $N_{\mathrm{H}}$ is within $<8 \times 10^{20} \mathrm{~cm}^{-2}$, which is therefore an upper limit of the systematic error due to the choice of the continuum. Using the LMC abundances (Table 2), we find column densities in the range of $(1.0-1.3) \times 10^{22} \mathrm{~cm}^{-2}$.

We detect a modulation of $N_{\mathrm{H}}$ with orbital phase. The observations $\mathrm{X} 1$ and $\mathrm{X} 2$ close to $\phi_{\text {orb }} \approx 0$, when the $\mathrm{BH}$ is behind the donor star, require a systematically higher $N_{\mathrm{H}}$ than $\mathrm{S} 3, \mathrm{C} 1$,

\footnotetext{
${ }_{4}$ As a convolution model that relies upon a spectral model outside of the energy range spanned by the noticed data, simpl must be evaluated on a suitably extended grid.

${ }^{5}$ For X1, the $N_{\mathrm{H}}$ derived with diskbb + compTT (Titarchuk 1994) is also consistent with the one from, e.g., simpl (diskbb).
}

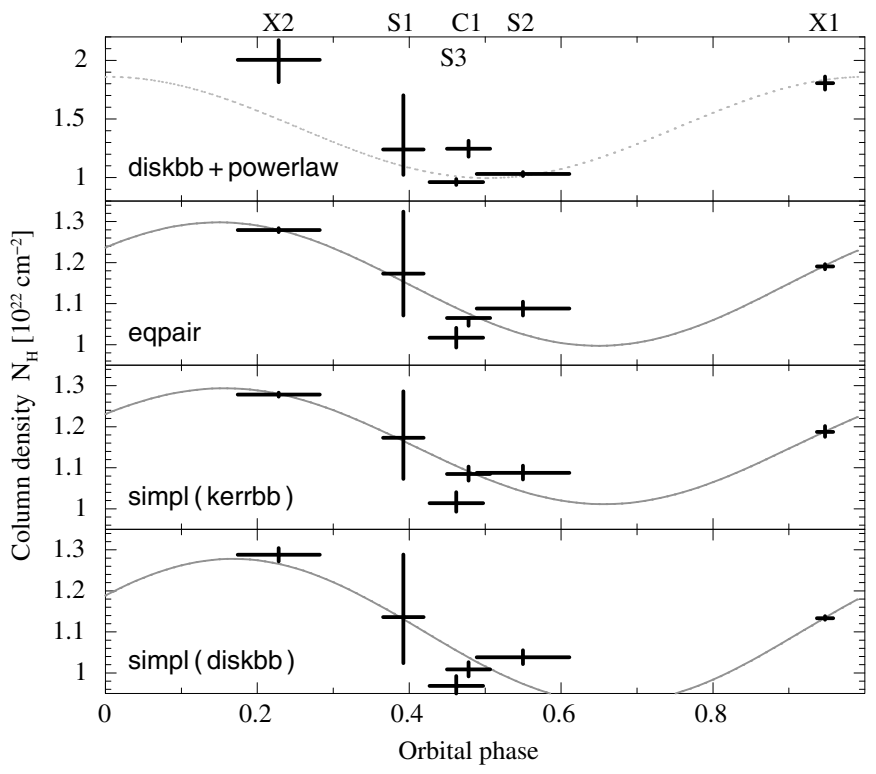

Fig. 3. $N_{\mathrm{H}}$ as a function of orbital phase $\phi_{\mathrm{orb}}^{(\mathrm{T} 3)}$ (see Table 1) using various continuum models. Note the different scale for the diskbb + powerlaw model, which may predict an unreliably large $N_{\mathrm{H}}$ (see text). The gray lines fit the results with sine curves.

and $\mathrm{S} 2$ close to $\phi_{\text {orb }} \approx 0.5$. To quantify this modulation by its mean and amplitude (Table 3), we fit sine curves to the six measurements for each continuum model (see Fig. 3), although we are aware that they do not describe the data very well and also predict the strongest absorption at $\phi_{\text {orb }}=0.15-0.17$, which is not expected.

Finally, we find marginal evidence of ionized absorption in the high-resolution spectra (Fig. 4), but a detailed study of these features is beyond the scope of this paper.

\section{Summary and discussion}

The elements with the largest contribution to the photoabsorption in the soft X-ray band are significantly less abundant in the 


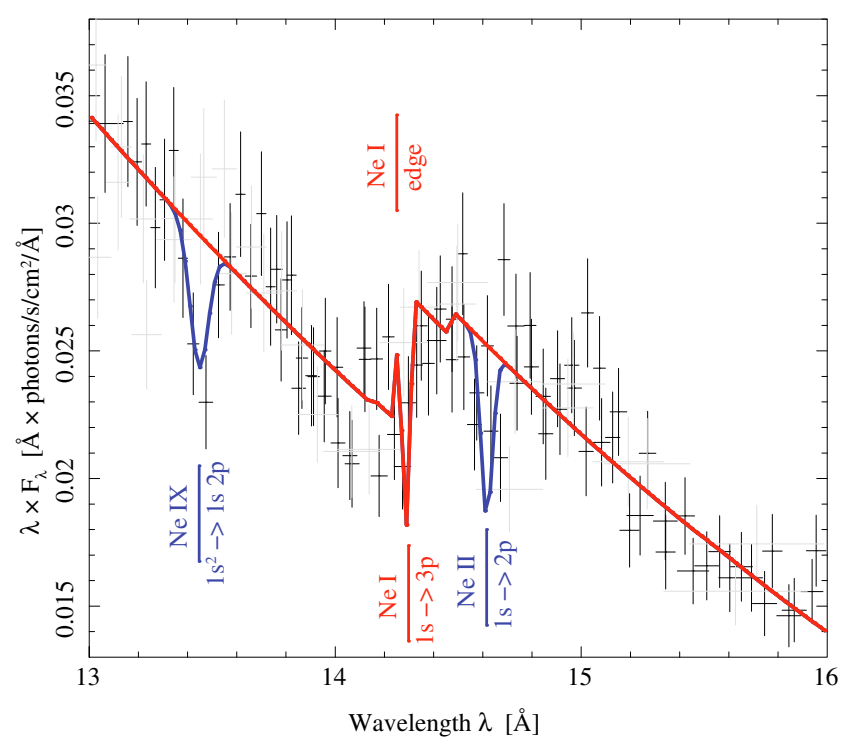

Fig. 4. The Ne-edge in observation X2. The first order RGS spectra (black) reveal absorption lines of Ne IX at $13.45 \AA$ and probably also $\mathrm{Ne}$ II at $13.62 \AA$, but the quality of the spectrum does not allow for a detailed study of the ionized absorber.

LMC than in the Galaxy (Table 2). Because of the lower metallicity, simply using radio-measured $N_{\mathrm{H}}$ values in an absorption model without adopting the LMC abundances will not allow for a correct description of the physical situation. Specifically for LMC X-1, the equivalent hydrogen column density inferred from this X-ray absorption study - taking the proper LMC abundances into account - is actually much higher than the H-column resolved by the LAB survey (at a half-power beam-width of 0.6 ; Kalberla et al. 2005), which is likely caused by additional material in the environment of LMC X-1 and in the system itself. This result was not obtained in earlier X-ray absorption measurements, as erroneously applying Galactic abundances resulted in lower $N_{\mathrm{H}}$ values.

In addition, we presented the first evidence that the column density varies in the range $(1.0-1.3) \times 10^{22} \mathrm{~cm}^{-2}$. A modulation with orbital phase is strongly suggested and would be consistent with absorption in the stellar wind of the donor giant. Orosz et al. (2009) assume that the orbital modulation of the $\mathrm{X}$-ray flux is mostly caused by Thomson scattering in the stellar wind since they find similar amplitudes ${ }^{6}$ in all three $R X T E$-ASM energy bands, namely $A_{\mathrm{A}(1.5-3 \mathrm{keV})}=7.2 \pm 1.0 \%, A_{\mathrm{B}(3-5 \mathrm{keV})}=$ $7.7 \pm 1.1 \%$, and $A_{\mathrm{C}(5-12 \mathrm{keV})}=3.8 \pm 2.9 \%$. From a modulation in $N_{\mathrm{H}}$ with a full amplitude of $3 \times 10^{21} \mathrm{~cm}^{-2}, A_{\mathrm{A}}=7.7-6.9 \%$, $A_{\mathrm{B}}=1.6-2.7 \%$, and $A_{\mathrm{C}}=0.4-1.7 \%$ are expected, depending on the assumptions about the ASM response; i.e., the variation seen with the ASM is almost consistent with the suggested neutral absorption. The phase of the current sine fit, however, is not. More soft X-ray observations covering more phases are clearly needed, because the structure of the stellar wind might be more complex than a sine curve. With the three $50 \mathrm{ks}$ Chandra observations that we have gained for AO 11, we will be able to better constrain the modulation.
Acknowledgements. We thank N. Przybilla and M. F. Nieva for providing the LMC abundances. M.H. and J.W. acknowledge funding from the Bundesministerium für Wirtschaft und Technologie through the Deutsches Zentrum für Luft- und Raumfahrt under contract 50OR0701. We thank the MIT Kavli Institute and the ISSI (Bern) for their hospitality during the preparation of this work.

\section{References}

Bajaja, E., Arnal, E. M., Larrarte, J. J., et al. 2005, A\&A, 440, 767 Burrows, D. N., Hill, J. E., Nousek, J. A., et al. 2005, SSR, 120, 165 Canizares, C. R., Davis, J. E., Dewey, D., et al. 2005, PASP, 117, 1144 Coppi, P. S. 2000, BAAS, 32, 1217

Cowley, A. P., Hutchings, J. B., \& Crampton, D. 1978, AJ, 83, 1619

Cowley, A. P., Schmidtke, P. C., Anderson, A. L., \& McGrath, T. K. 1995, PASP, 107,145

Cui, W., Feng, Y. X., Zhang, S. N., et al. 2002, ApJ, 576, 357

den Herder, J. W., Brinkman, A. C., Kahn, S. M., et al. 2001, A\&A, 365, L7

Done, C., Życki, P. T., \& Smith, D. A. 2002, MNRAS, 331, 453

Dufour, R. J. 1984, in Structure and Evolution of the Magellanic Clouds, ed. S. van den Bergh, \& K. S. D. Boer, IAU Symp., 108, 353

Ebisawa, K., Mitsuda, K., \& Inoue, H. 1989, PASJ, 41, 519

Garnett, D. R. 1999, in New Views of the Magellanic Clouds, ed. Y. H. Chu, N. Suntzeff, J. Hesser, \& D. Bohlender, IAU Symp., 190, 266

Gierliński, M., Maciołek-Niedźwiecki, A., \& Ebisawa, K. 2001, MNRAS, 325, 1253

Gou, L., McClintock, J. E., Liu, J., et al. 2009, ApJ, 701, 1076

Haardt, F., Galli, M. R., Treves, A., et al. 2001, ApJS, 133, 187

Houck, J. C., \& Denicola, L. A. 2000, in Astronomical Data Analysis Software and Systems IX, ed. N. Manset, C. Veillet, \& D. Crabtree, ASP Conf. Ser., 216,591

Hutchings, J. B., Crampton, D., \& Cowley, A. P. 1983, ApJ, 275, L43

Hutchings, J. B., Crampton, D., Cowley, A. P., et al. 1987, AJ, 94, 340

Jansen, F., Lumb, D., Altieri, B., et al. 2001, A\&A, 365, L1

Kalberla, P. M. W., Burton, W. B., Hartmann, D., et al. 2005, A\&A, 440, 775

Korn, A. J., Keller, S. C., Kaufer, A., et al. 2002, A\&A, 385, 143

Korn, A. J., Nieva, M. F., Daflon, S., \& Cunha, K. 2005, ApJ, 633, 899

Levine, A. M., \& Corbet, R. 2006, ATel, 940

Li, L. X., Zimmerman, E. R., Narayan, R., \& McClintock, J. E. 2005, ApJS, 157, 335

Mark, H., Price, R., Rodrigues, R., et al. 1969, ApJ, 155, L143

Negueruela, I., \& Coe, M. J. 2002, A\&A, 385, 517

Noble, M. S., \& Nowak, M. A. 2008, PASP, 120, 821

Noble, M. S., Houck, J. C., Davis, J. E., et al. 2006, in Astronomical Data Analysis Software and Systems XV, ed. C. Gabriel, C. Arviset, D. Ponz, \& S. Enrique, ASP Conf. Ser., 351, 481

Nowak, M. A., Wilms, J., Heindl, W. A., et al. 2001, MNRAS, 320, 316

Orosz, J. A., Steeghs, D., McClintock, J. E., et al. 2009, ApJ, 697, 573

Pakull, M. 1980, IAU Circ., 3472, 1

Price, R. E., Groves, D. J., Rodrigues, R. M., et al. 1971, ApJ, 168, L7

Przybilla, N., Nieva, M. F., Heber, U., et al. 2008, A\&A, 480, L37

Richter, O. G., Tammann, G. A., \& Huchtmeier, W. K. 1987, A\&A, 171, 33

Schlegel, E. M., Marshall, F. E., Mushotzky, R. F., et al. 1994, ApJ, 422, 243

Shrader, C., \& Titarchuk, L. 1998, ApJ, 499, L31

Smith, D. M., Dawson, D. M., \& Swank, J. H. 2007, ApJ, 669, 1138

Steiner, J. F., Narayan, R., McClintock, J. E., \& Ebisawa, K. 2009, PASP, 121, 1279

Strüder, L., Briel, U., Dennerl, K., et al. 2001, A\&A, 365, L18

Suchy, S., Pottschmidt, K., Wilms, J., et al. 2008, ApJ, 675, 1487

Titarchuk, L. 1994, ApJ, 434, 570

Turner, M. J. L., Abbey, A., Arnaud, M., et al. 2001, A\&A, 365, L27

Wilms, J., Allen, A., \& McCray, R. 2000, ApJ, 542, 914

Wilms, J., Nowak, M. A., Pottschmidt, K., et al. 2001, MNRAS, 320, 327

Wilms, J., Nowak, M. A., Pottschmidt, K., et al. 2003, in New Views on

Microquasars, ed. P. Durouchoux, Y. Fuchs, \& J. Rodriguez, 49

Wilms, J., Nowak, M. A., Pottschmidt, K., et al. 2006, A\&A, 447, 245

Yao, Y., Wang, Q. D., \& Nan Zhang, S. 2005, MNRAS, 362, 229

$\overline{6}$ The fractional full amplitude is here $A=(\max -\min ) /$ mean. 